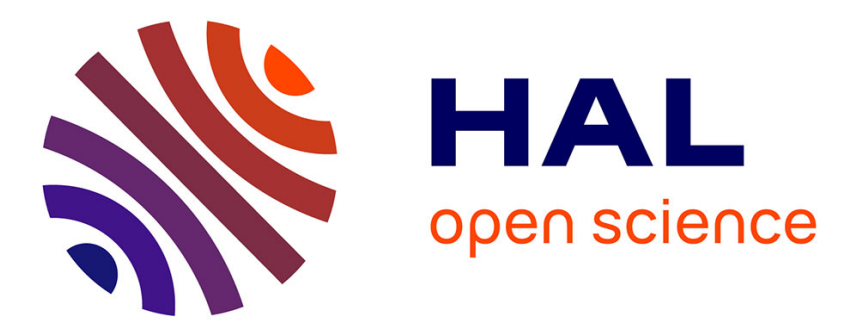

\title{
Modal Overlap Factor of a beam with an acoustic black hole termination
}

\author{
Vivien Denis, Adrien Pelat, François Gautier, Benjamin Elie
}

\section{To cite this version:}

Vivien Denis, Adrien Pelat, François Gautier, Benjamin Elie. Modal Overlap Factor of a beam with an acoustic black hole termination. Journal of Sound and Vibration, 2014, 333 (12), pp.2475-2488. 10.1016/j.jsv.2014.02.005 . hal-01288274

\section{HAL Id: hal-01288274 https://hal.science/hal-01288274}

Submitted on 14 Mar 2016

HAL is a multi-disciplinary open access archive for the deposit and dissemination of scientific research documents, whether they are published or not. The documents may come from teaching and research institutions in France or abroad, or from public or private research centers.
L'archive ouverte pluridisciplinaire HAL, est destinée au dépôt et à la diffusion de documents scientifiques de niveau recherche, publiés ou non, émanant des établissements d'enseignement et de recherche français ou étrangers, des laboratoires publics ou privés. 


\title{
Modal Overlap Factor of a beam with an Acoustic Black Hole termination
}

\author{
V. Denis ${ }^{1}$, A. Pelat ${ }^{1}$, F. Gautier ${ }^{1}$, B. Elie $^{1}$ \\ Laboratoire d'Acoustique de l'Université du Maine, Avenue Olivier Messian, 72085 Le \\ Mans, Cedex 09
}

\begin{abstract}
Acoustic Black Hole (ABH) effect is a passive vibration reduction technique which takes advantage of properties of wave propagation in thin structures of varying thickness. A practical implementation of $\mathrm{ABH}$ on a uniform beam consists in an extremity whose thickness follows a power-law profile covered by a very thin layer of additional damping material. A modal analysis based on a High Resolution technique shows that the ABH significantly increases the Modal Overlap Factor (MOF) of the beam, thus reducing the resonant behaviour of the structure. Further investigations, including a two dimensional numerical model of the structure based on the finite difference method, show that this MOF can be explained by an increase of the modal density and a high damping of a number of modes of the structure due to the $\mathrm{ABH}$.
\end{abstract} Keywords:

flexural vibration, damping, modal analysis, acoustic black hole, passive damping technique

Email address: vivien.denis@univ-lemans.fr (V. Denis) 


\section{Introduction}

Vibration damping of mechanical structures such as beams and plates is playing an important role in the design of many industrial applications. For reducing structural vibrations, classical methods consist in covering the 5 structure with a heavy visco-elastic material. The efficiency of these methods has been shown and widely studied $[1,2]$ but they result in a strong added mass on the treated structure, which may be prohibitive in transportation industry for ecological and economical reasons. Then, the development of vibration damping methods without added mass becomes of a great interest.

In the case of bending waves in thin structures, the vibrating behaviour at one point of the structure is well represented by the driving mechanical admittance, also called driving mobility and defined as the ratio between the vibration velocity and the input exciting force. When represented as a function of the frequency, the driving mobility displays resonance and antiresonance peaks around a mean value. In the framework of the Kirchhoff theory [3], the mean value corresponds to the mobility of an infinite plate and depends on the structure material and thickness [4]. The frequency, magnitude and loss factor of the resonance peaks depend both on the size and material of the structure, and on the boundary conditions. Hence, for given structure and material, attenuating bending wave reflections at the edges is a way to reduce the resonant behaviour which can be responsible for structure damages.

To achieve this issue, Vemula et al. [5] proposes a method by using a 
graded impedance interface at the edges of a beam, consisting in the association of several pieces of beams made with different materials. The results show that lower reflectivity is caused by energy dissipation within the composite material at the free end coupled with relatively large amplitude vibrations caused by the impedance gradation. Another method consists in taking advantage of an Acoustic Black Hole (ABH) effect $[6,7,8]$. The ABH effect is related to bending wave properties in a beam of decreasing thickness: Mironov [9] shows that at the neighbourhood of the edge, if the thickness decreases smoothly to zero, the wave slows down and stops without being reflected at the edge. The condition of sufficient smoothness can be fulfilled by a power-law thickness profile $h(x)$ in the form

$$
h(x)=\epsilon x^{m},
$$

where $m \geq 2$ (see Fig. 5). If the thickness is strictly zero at the edge [9], it can be shown that the needed travel time for a wave to reach the edge becomes infinite. Thus, the reflection coefficient tends to zero. Despite this promising theory, manufacturing processes are such as the truncation of the tapered profile can never be small enough for the effect to be attractive.

However, by covering the tapered profile by a thin damping layer, Krylov $[6,7]$ shows, in the framework of geometrical acoustics, that the damping layer compensates the finite thickness at the edge of practical structures. He obtains an analytical expression of the reflection coefficient which is drastically reduced compared to its value for a free extremity. A more complete model that takes into account the effect of evanescent waves in one dimensional structures has been proposed by Georgiev et al. [8]. In this model 

to compute a mechanical impedance matrix along the tapered beam and derive a reflection matrix. The results notably show that some rules can be specified for determining the optimal geometrical and material properties of the damping layer [10]. The first circular ABH has been proposed by Gautier circular plate has been proposed by O'boy et al. [14].

Let us introduce the subject by presenting the efficiency of $\mathrm{ABH}$ for vibration damping. Fig. 1(a) shows such circular ABH included in an aluminium polygonal plate of thickness $1.5 \times 10^{-3} \mathrm{~m}$ (dimensions are indicated on Fig. 1(a)). Fig. 1(b) shows the frequency response functions of this polygonal plate with and without the $\mathrm{ABH}$ treatment. It can clearly be seen that $\mathrm{ABH}$ brings an important smoothing of the response curve, leading to a reduction of the resonant behaviour of the plate from $2000 \mathrm{~Hz}$. Characterizabut it only has a phenomenological interest and does not provide more physical explanations. In the two cases, this response is complex because of the high modal density. Traditional modal approaches fail to correctly analyse such structures at high frequencies mainly because of the number of resonances and a high modal overlap [15]. Mid and high frequency techniques however are able to give useful informations and can be used to characterize the ABH effect on plate-like structures. A first step is however to study this $\mathrm{ABH}$ effect in a one dimensional academic configuration, with techniques that are appropriate for more complex structures. 
[Figure 1 about here.]

The current article deals with the implementation of the $\mathrm{ABH}$ effect on beams and shows both experimentally and numerically that it can be seen as a way to increase the modal overlap factor of the structure, therefore reducing its resonant behaviour. In Sec. 2, the damping produced by the $\mathrm{ABH}$ effect is experimentally evaluated through estimations of the modal parameters obtained from mobility measurements that are post processed by using a high resolution modal identification technique. Sec. 3 presents a numerical finite difference model that is correlated with the experimental results. In Sec. 4 the results, in terms of modal shapes and modal damping of the eigenmodes, provide a good understanding of how the $\mathrm{ABH}$ makes the modal overlap increase. Sec. 5 is dedicated to the comparison between numerical and experimental results. Some conclusions are drawn in Sec. 6 .

\section{Experimental modal analysis of an $\mathrm{ABH}$ beam}

In this section, experimental results obtained on beams made of aluminium, whose dimensions are those appearing in Tab. 1, are presented and illustrate the smoothing of the mobility curve induced by the $\mathrm{ABH}$ effect. A reference beam of uniform thickness is compared with a treated beam having at one of its extremities an $\mathrm{ABH}$ profile. On the $\mathrm{ABH}$ beam, part of the tapered profile is covered by a thin layer of a damping material (see Fig. 2(a)). 
[Table 1 about here.]

\subsection{Experimental setup}

Fig. 2(b) represents the experimental setup in which the sample to be tested is vertically suspended. To avoid unwanted additional damping caused by the suspensions, thin wires are glued to the side ridges of the beam. An miniature impact hammer (PCB Piezotronics 086E80) mounted on a flexible support excites the beam at one arbitrary point of its centerline in order to mainly excite flexural modes and to avoid torsional modes. A force sensor is embedded in the hammer and measures the impact force. At the same point, but on the back side of the beam, the measurement of acceleration of the beam is made by a miniature accelerometer (PCB Piezotronics 352C23, $0.3 \mathrm{~g})$. The time signals are recorded by using a National Instrument NI USB-4431 acquisition card. The experimental set up is driven by a Matlab ${ }^{\circledR}$ application which also performs classical signal post processing operations.

[Figure 2 about here.]

[Figure 3 about here.]

Fig. 3 presents the frequency response functions for the two structures. While the acoustic black hole effect plays the same role in this case than in its implementation in plates (see Fig. 1(b)), the curves clearly show a low frequency behaviour that can be studied using traditional modal analysis techniques. However, techniques usually applied to high frequency behaviour are introduced in this article to allow further studies on plates. 


\subsection{High resolution modal identification techniques}

Since the modal overlap is expected to be large in the case of platelike structures, classic modal identification techniques may be no longer robust $[15,16]$. Subspace methods like MUSIC (MUltiple SIgnal Classification) [17], Matrix Pencil [18], or ESPRIT (Estimation of Signal Parameters via Rotational Invariance Techniques) [19] are then interesting to consider and have already been successfully applied to vibration signals [20, 21, 22]. Indeed, these methods overcome the Fourier resolution limit and are then useful when modes are overlapping, hence the designation high-resolution. In this work, ESPRIT is used since it is known to be one of the more robust.

\subsubsection{ESPRIT algorithm}

The velocity response $s(A, t)$ in any point $A$ to an impulse force is modelled as the real part of a sum of complex damped sinusoids, each one being the time response of a mode:

$$
s(A, t)=\operatorname{Re}\left[\sum_{k=1}^{K} b_{k}(A) z_{k}^{t}\right],
$$

where $b_{k}(A)$ is the complex amplitude of the $k$-th mode and $z_{k}=\mathrm{e}^{-a_{k}+\mathrm{j} \omega_{d k}}$ denotes the corresponding pole with modal damped pulsation $\omega_{d k}$ and modal damping factor $a_{k}$. $K$ is the number of complex poles in the frequency band $\left[-F_{s} / 2 \quad F_{s} / 2\right]$, where $F_{s}$ is the sampling frequency.

The ESPRIT [19] algorithm estimates the signal parameters corresponding to the modal parameters of the $K$ sinusoidal components embedded in the 
signal. Like other subspace high resolution methods (such as Matrix Pencil or MUSIC), it is based on the decomposition of the data vector space onto two orthogonal subspaces, the so-called signal and noise subspaces. Then, using the so-called rotational invariance property of the signal subspace (it remains invariant from a sample to the next), the poles $z_{k}$ spanning the signal subspace can be estimated accurately. The reader can refer to Ref. [22] for a detailed description of the ESPRIT algorithm. The subspace decomposition and the use of the rotational invariance property make the ESPRIT method more robust and accurate than classic Prony-based method, such as LSCE [23] for instance.

\subsubsection{Signal enumeration technique}

The estimation of the model order $K$, related to the number of modes in the frequency band of analysis, is performed thanks to the signal enumeration technique ESTER (ESTimation of ERror), designed by Badeau et al. [24] and used by Ege [21] and Elie et al. [22] in the context of musical acoustics. The ESTER criterion has been chosen among other signal enumeration techniques $[25,26,27,28,29,30]$ for its reliability and also for its straightforward implementation with the ESPRIT algorithm.

This criterion consists in appraising the rotational invariance property of the signal subspace with an error function. This latter being minimal when the rotational invariance property is verified, namely when the modelling order is equal to the right number of sinusoidal components. Note that for impulse responses signal, which is real, the number of components $K$ is twice 
the number of physical eigenmodes.

\subsection{Effect of the $A B H$ on modal parameters}

Modal parameters are estimated by applying the ESPRIT technique described in section 2.2.1 on the driving point mobility shown in Fig. 3. Fig. 4 presents a comparison of the experimental modal parameters between the reference beam $(\square)$ and the ABH beam (o).

On Fig. 4(a), the modal density of the reference beam fits very well with a theoretical prediction given by the Euler-Bernoulli beam theory [31]. These two results are in good agreement since particular attention has been paid to excite and measure the beam on its centerline: the vibrational field is almost entirely carried by the flexural modes, while the torsional modes only provide a very weak contribution. Fig. 4(a) also shows that the ABH termination used in this experiment does not lead to a substantial increase of the modal density. The modal density of the $\mathrm{ABH}$ beam is very similar to the one of the reference beam: in fact, it is very slightly higher as it will be explained in Sec. 4.

An important increase of the modal loss factors is seen on Fig. 4(b) for the ABH case. Those of the reference beam, of uniform thickness and material properties, are almost constant against the frequency (i.e. independent of the mode) and are equal to approximately $0.2 \%$. This expected weak constant value corresponds to the intrinsic material loss factor of the beam (aluminium). The modal loss factors for the ABH beam are at least ten times 
higher than those of the reference beam and can reach about $4 \%$.

The Modal Overlap Factor (MOF) is the ratio between the modal bandwidth at the half amplitude and the average modal spacing [15]. The MOF can also be calculated as the product $[21,16]$

$$
\mathrm{MOF}=d \eta f
$$

where $d$ is the modal density, $\eta$ is the modal loss factor and $f$ is the frequency. In structural dynamics, the MOF can be used to establish three frequency ranges : the low frequency range $(\mathrm{MOF}<30 \%)$ in which the structure admits a resonant behaviour, the mid frequency range $(30 \%<\mathrm{MOF}<100 \%)$ in which the modal overlap and/or loss factor are higher, and the high frequency range $(\mathrm{MOF}>100 \%)$ in which diffuse field assumptions are reached. Diffuse field may easily be obtained in plates; this is not the case for the beams presented in this paper.

Fig. 4(c) shows that the $\mathrm{ABH}$ termination results in an increase of the MOF: while it barely reaches $5 \%$ for the reference beam, it is more than ten times higher and can reach $50 \%$ for the $\mathrm{ABH}$ beam above a starting frequency of efficiency (about $400 \mathrm{~Hz}$ ). This strong increase of the MOF is directly related to the increase of the modal loss factors.

Finally, Fig. 4 shows that the result on the mobility seen in Fig. 3 corresponds to an important increase of the MOF which is essentially due to the $\mathrm{ABH}$ action on the modal loss factor. This result means that the $\mathrm{ABH}$ makes the structure vibrate in a frequency range intrinsically more damped. 
The reasons for this important increase are studied in Sec. 4, thanks to the numerical model developed in Sec. 3.

[Figure 4 about here.]

\section{Finite difference model of the $\mathrm{ABH}$ beam}

To give better understanding of the experimental results, a model of flexural vibrations in a non uniform thin plate is proposed. A plate model is considered instead of a beam one because the expected flexural wavelength at the end of the $\mathrm{ABH}$ extremity reaches down the width of the cross-section in the frequency band studied. The governing equations of such a model are given in Sec. 3.1 and a numerical resolution from a finite difference scheme adapted to the ABH profile is then described in Sec. 3.2. All the variables refer to the scheme on Fig. 5.

[Figure 5 about here.]

\subsection{Governing equations}

The equation of the free transverse vibration $W(x, y)$ of the structure in the case of an harmonic motion is given by Ref. [32]:

$\nabla^{2}\left(D(x) \nabla^{2} W(x, y)\right)-(1-\nu)\left(\frac{\partial^{2} D(x)}{\partial x^{2}} \frac{\partial^{2} W(x, y)}{\partial y^{2}}\right)-\rho(x) h(x) \omega^{2} W(x, y)=0$

where $\rho(x)$ is the mass density, $h(x)$ is the thickness, $\omega$ is the angular frequency, $D(x)$ is the local complex bending stiffness and $\nu$ the Poisson co-

efficient. Coordinates $x$ and $y$ are defined in Fig. 5. In Eq. 4, the bending 
stiffness, thickness and mass density are characteristic of the sandwich structure composed by the association of the aluminium beam and the damping layer.

The model uses structural damping, represented with a loss factor included in the imaginary part of the complex bending stiffness $D(x)$ in Eq. 4 . The effect of the layer is represented with equivalent mechanical properties that modify the bending stiffness, the mass density and the thickness, following the model of Ross-Ungar-Kerwin [1] as used in the current context by Georgiev [8]. The complex bending stiffness is thus given by

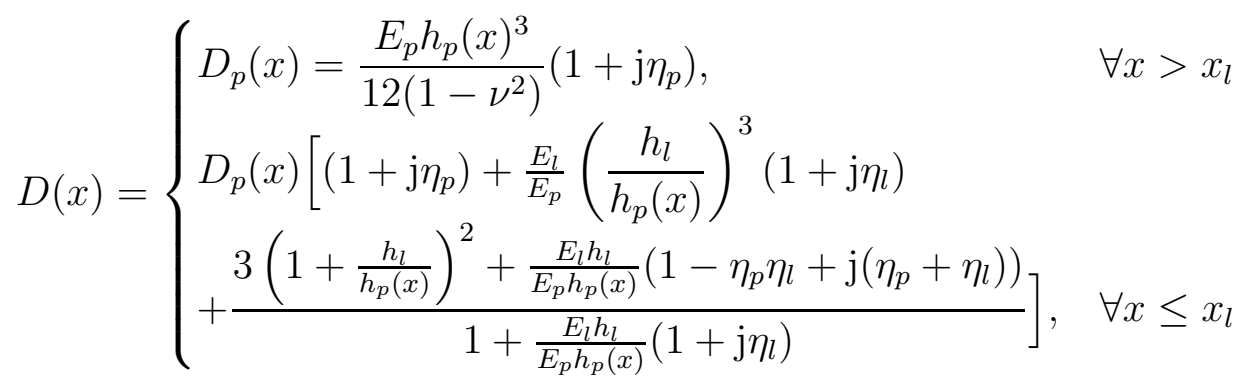

where $D_{p}(x), E_{p}, \eta_{p}$ and $h_{p}(x)$ are the bending stiffness, the Young modulus, the loss factor and the thickness of the plate alone, respectively , $E_{l}, \eta_{l}$ and $h_{l}$ are the Young modulus, the loss factor and the thickness of the damping layer, respectively. One must also take into account the effect of the mass of the layer and its thickness using an equivalent mass density $\rho(x)$ :

$$
\rho(x)= \begin{cases}\rho_{p}, & \forall x>x_{l} \\ \frac{\rho_{p} h_{p}(x)+\rho_{l} h_{l}}{h_{p}(x)+h_{l}}, & \forall x \leq x_{l}\end{cases}
$$

where $\rho_{p}$ and $\rho_{l}$ are the mass densities of the structure and the layer, respec- 
tively, and a total thickness $h(x)$ of the sandwich plate:

$$
h(x)= \begin{cases}h_{p}(x), & \forall x>x_{l} \\ h_{p}(x)+h_{l}, & \forall x \leq x_{l},\end{cases}
$$

where the thickness of the plate is, in the ABH case,

$$
h_{p}(x)= \begin{cases}h_{0}, & \forall x>x_{\mathrm{ABH}} \\ h_{0} \frac{\left(x+x_{\mathrm{ABH}}\right)^{m}}{\left(x_{0}+x_{\mathrm{ABH}}\right)^{m}}, & \forall x \leq x_{\mathrm{ABH}} .\end{cases}
$$

The truncation thickness $h_{t}$ (see Fig. 5) is the thickness at the very tip of the ABH profile $(x=0)$.

Free boundary conditions are considered at the ABH extremity $(x=0)$, the other beam extremity $(x=L)$ and on lateral edges $(y=0$ and $y=b)$. The so-called Kelvin-Kirchhoff edge reaction and the bending moment are zero at the edges and can be derived to obtain, respectively,

$$
\begin{gathered}
V_{x}=-\frac{\partial}{\partial x}\left(D\left(\frac{\partial^{2} W}{\partial x^{2}}+\nu \frac{\partial^{2} W}{\partial y^{2}}\right)\right)-2 D(1-\nu) \frac{\partial^{3} W}{\partial x \partial y^{2}}=0 \quad \text { for } x=0, L \quad \forall y, \\
M_{x}=-D\left(\frac{\partial^{2} W}{\partial x^{2}}+\nu \frac{\partial^{2} W}{\partial y^{2}}\right)=0 \quad \text { for } x=0, L \quad \forall y,
\end{gathered}
$$

along the $y$-direction, and

$$
\begin{gathered}
\left.V_{y}=-D \frac{\partial}{\partial y}\left(\frac{\partial^{2} W}{\partial y^{2}}+\nu \frac{\partial^{2} W}{\partial x^{2}}\right)\right)-2(1-\nu) \frac{\partial}{\partial x}\left(D \frac{\partial^{2} W}{\partial x \partial y}\right)=0 \quad \text { for } y=0, b \quad \forall x, \\
M_{y}=-D\left(\frac{\partial^{2} W}{\partial y^{2}}+\nu \frac{\partial^{2} W}{\partial x^{2}}\right)=0 \quad \text { for } y=0, b \quad \forall x,
\end{gathered}
$$

along the $\mathrm{x}$-direction. Free boundary conditions also require [32] that

$$
M_{x y}=-D(1-\nu) \frac{\partial^{2} W}{\partial x \partial y}=0 \quad \text { at points }(0,0),(0, b),(L, 0) \text { and }(L, b) \text {. }
$$



problem, that can be solved according to the numerical method described in Sec. 3.2.

A discussion on the validity of the model is relevant at this point. It is emphasized that the dispersion relation is such that at a given frequency, if the wavelength is greater than the thickness in the uniform part of the plate, it is also the case in the tapered zone. Therefore, since the classical theory of plate based on the Kirchhoff model is valid in the region of uniform thickness, it also applies in the tapered zone and shear and rotary inertia are expected to be negligible. One is also reminded that the model does not take into account some characteristics of the practical realisation of the $\mathrm{ABH}$ effect: the lack of symmetry and added damping may move the neutral plane of the structure and generate plane extension behaviour that is coupled with flexural behaviour. According to the literature $[6,8,9]$, flexural behaviour seems sufficient to produce the $\mathrm{ABH}$ effect and may therefore be the dominant mechanism. Other effects are thus neglected in this paper but may be investigated in a further study.

\subsection{Numerical scheme}

The problem described in Sec. 3.1 is numerically solved by using a secondorder finite difference scheme $[33,34,35]$. The spatial derivative operators in Eq. 4 and Eqs. 9-13 can be approximated from Taylor series. To introduce the needed notations, a discrete approximation by backward finite difference 
of the first derivative of the function $W(x)$ is given as an example:

$$
\frac{\mathrm{d} W(x)}{\mathrm{d} x} \approx \frac{1}{\Delta_{x}}\left(w_{n}-w_{n-1}\right)=\delta_{-} w_{n}
$$

where $w_{n}=W(x), x=n \Delta_{x}$ with $\Delta_{x}$ the spatial step and $n$ the spatial index, and $\delta_{-}$is the backward differential operator. A backward spatial averaging operator $\mu_{-}$defined such that $\mu_{-} w_{n}=\left(w_{n}+w_{n-1}\right) / 2$, is also applied for the numerical scheme suitability.

In classical cases for which the thickness of the plate is constant, such a second-order finite difference scheme is straightforward. The present case is more difficult since the thickness is locally reduced, leading to an important numerical dispersion or an important computation cost. Indeed, the wavelength in the $\mathrm{ABH}$ structure dramatically decreases along the $\mathrm{ABH}$ profile, since it depends on the local thickness for a given frequency. To correctly represent very small wavelengths and to avoid numerical dispersion, a non uniform mesh grid is proposed. A practical implementation consists in using a coordinate change that switches a non-uniform "physical" mesh grid into a transformed uniform mesh grid where a transformed equation of motion is solved [34]. The interest is to avoid a tedious resolution on a non-uniform grid. Variable $x$ defines the physical coordinate and variable $\tilde{x}$ defines the transformed coordinate. Variable $\lambda=2 \pi \sqrt[4]{E h^{2} / 12 \rho\left(1-\nu^{2}\right) \omega^{2}}$ represents the wavelength in the physical space whereas variable $\tilde{\lambda}$ is the wavelength in the transformed space. The coordinate change may be chosen in order to match the change in wavelength so that

$$
\frac{\lambda(x)}{\mathrm{d} x}=\frac{\tilde{\lambda}}{\mathrm{d} \tilde{x}}
$$



ing to classical plate theory [32], the coordinate change is chosen as follows:

$$
\mathrm{d} \tilde{x}=\frac{\mathrm{d} x}{\tilde{x}_{\mathrm{av}} \sqrt{h(x)}} \quad \text { with } \quad \tilde{x}_{\mathrm{av}}=\int_{0}^{L} \frac{1}{\sqrt{h(x)}} \mathrm{d} x
$$

where $L$ is the dimension of the plate along the $x$-direction.

Note that no coordinate change is applied to the $y$ coordinate. The equation to solve on the transformed grid, derived from Eq. 4, is finally the following:

$$
\begin{gathered}
\frac{h_{n}^{-1 / 2}}{\tilde{x}_{\mathrm{av}}^{2}} \delta_{\tilde{x}+}\left[\left(\mu_{\tilde{x}-} h_{n}^{-1 / 2}\right) \delta_{\tilde{x}-}\left[D_{n}\left(\frac{h_{n}^{-1 / 2}}{\tilde{x}_{\mathrm{av}}^{2}} \delta_{\tilde{x}+}\left(\left(\mu_{\tilde{x}-} h_{n}^{-1 / 2}\right) \delta_{\tilde{x}-} w_{n, q}\right)+\delta_{y y} w_{n, q}\right)\right]\right] \\
+D_{n} \delta_{y y}\left[\frac{h_{n}^{-1 / 2}}{\tilde{x}_{\mathrm{av}}^{2}} \delta_{\tilde{x}+}\left(\left(\mu_{\tilde{x}-} h_{n}^{-1 / 2}\right) \delta_{\tilde{x}-} w_{n, q}\right)+\delta_{y y} w_{n, q}\right] \\
-(1-\nu)\left(\frac{h_{n}^{-1 / 2}}{\tilde{x}_{\mathrm{av}}^{2}} \delta_{\tilde{x}+}\left(\left(\mu_{\tilde{x}-} h_{n}^{-1 / 2}\right) \delta_{\tilde{x}-} D_{n}\right) \delta_{y y} w_{n, q}\right)-\rho h_{n} \omega^{2} w_{n, q}=0 .
\end{gathered}
$$

where $n$ and $q$ are the spatial indexes of the grid related to $\tilde{x}$ and $y$ respectively, $\delta_{\tilde{x}-}$ and $\delta_{\tilde{x}+}$ are respectively the backward and forward finite difference operators, $\mu_{\tilde{x}-}$ and $\mu_{\tilde{x}+}$ are respectively the backward and forward averaging operators and $\delta_{y y}$ is the approximation to the second derivative in $y$ [34]. The transformed boundary conditions are also derived from finite difference operators [34] but they will not be shown here for the sake of conciseness.

The eigenproblem of Eq. 17 can be written under matrix formulation:

$$
\left(\mathbf{K}-\alpha^{2} \mathbf{M}\right) \boldsymbol{\phi}=0
$$

where $\mathbf{K}$ and $\mathbf{M}$ are respectively the complex stiffness matrix and the mass matrix of the structure, $\alpha$ is an eigenvalue and $\boldsymbol{\phi}$ is an eigenvector. 


\section{Numerical modal analysis}

Numerical modal analysis is performed for a configuration that matches the material and geometrical parameters of the experimental sample. The grid is constituted of $320 \times 120$ elements and attention is paid to the spatial step along the y-axis that should be small enough to correctly represent the small wavelengths at the ABH extremity. Only symmetric modes about the centerline are considered in the post-processing of the results so that the torsional modes, which are not excited in the experiment, are eliminated. All the set values of the parameters are listed in Tab. 1 and refer to Fig. 5.

The values for parameters $E_{l}$ and $\eta_{l}$ (appearing in Tab. 1) are chosen to obtain a good agreement on the MOF (see Fig. 12) between simulation and experience and are in accordance with the literature concerning this type of material [36].

\subsection{Study of the spectrum of the complex eigenvalues}

\subsubsection{Typical results}

According to the literature [3], the eigenvalues $\alpha_{k}$ of the problem defined in Eq. 18 can be written:

$$
\alpha_{k}=-\omega_{k}\left(\xi_{k}-\mathrm{j} \sqrt{1-\xi_{k}^{2}}\right)
$$

where $\omega_{k}$ are the natural frequencies and $\xi_{k}$ are the modal damping ratios (with $\eta_{k}=2 \xi_{k}$ the modal loss factors). 
Fig. 6(a) displays the thickness profile of the reference beam $(\Delta)$ and the $\mathrm{ABH}$ beam $(\nabla)$. The eigenvalues for these two cases are plotted in the complex plane on Fig. 6(b). For any mode $k$ with eigenvalue $\alpha_{k}$ of coordinates $\left(-\operatorname{Re}\left(\alpha_{k}\right), \operatorname{Im}\left(\alpha_{k}\right)\right)$ on Fig. 6(b), the modal loss factor of this mode is related to the angle between the imaginary axis and the direction $\left((0,0),\left(-\operatorname{Re}\left(\alpha_{k}\right), \operatorname{Im}\left(\alpha_{k}\right)\right)\right)$ so that an increase of this angle is simply an increase of the modal loss factor.

It is shown that most of the eigenvalues of the $\mathrm{ABH}$ are located along a straight line in the complex plane. The associated modes have then a loss factor that is roughly close to a constant value and that is higher than that of the reference: $\mathrm{ABH}$ effect leads to an increase of the modal loss factor for the majority of the modes. This is the case of modes 14 and 42 indicated on Fig. 6(b), as examples. This phenomenon can be more clearly viewed on Fig. 7 where are plotted the modal loss factors for the two cases in the frequency range $[0-4000 \mathrm{~Hz}]$. On this figure, small variations around $2 \%$ can be observed in the $\mathrm{ABH}$ case.

Some eigenvalues of $\mathrm{ABH}$ do not belong to the same line and have a higher real part. The associated modes have then a greater loss factor. Examples of modes 23 and 41 are indicated on Fig. 6(b). They can conveniently be called "hyper-damped" modes.

[Figure 6 about here.] 
The inhomogeneity of the mechanical properties all over the domain has some consequences on the eigenfrequencies and the eigenmodes of the structure. In a conservative case, $D_{p}(x)$ is real and the problem has purely imaginary eigenvalues $\alpha_{k}$ and purely real eigenmodes $\phi_{k}$. In a dissipative case where the dissipation is homogeneous, the bending stiffness can be written $D_{p}(x)=E_{p} h_{p}^{3}(x) / 12\left(1-\nu^{2}\right) \times\left(1+\mathrm{j} \eta_{p}\right)$ where $\eta_{p}$ is constant, and $\left(1+\mathrm{j} \eta_{p}\right)$ can get out of the spatial operator in Eq. 4; the eigenmodes are real and are the same than in the conservative case; the eigenvalues however are multiplied by a complex number depending on the material loss factor only [3]. This implies that the loss factor of a uniform structure and a non-uniform structure are the same if they are equally and homogeneously damped, even if their natural frequencies are different. In a dissipative case where the dissipation is non homogeneous over the space, both eigenvalues and modes are complex and different from the conservative or dissipative with homogeneous dissipation cases. This means that the increase of loss factor seen on Fig. 6(b) and Fig. 7 comes from the spatial repartition of the losses. It puts the light on the role of the damping layer: without it, the damping properties are homogeneous and the loss factors are the same than in the reference case.

\subsubsection{Efficiency threshold of the $A B H$}

Fig. 7 shows the modal loss factors on the frequency range $[0-4000 \mathrm{~Hz}]$ and allows to see an efficiency threshold in frequency for the $\mathrm{ABH}$ : in the simulated case, below $200 \mathrm{~Hz}$ modal loss factors of the $\mathrm{ABH}$ beam and the reference beam have the same order of magnitude. From $250 \mathrm{~Hz}$, the loss factors of the $\mathrm{ABH}$ beam suddenly increase to reach a value of about $2 \%$ in 
[Figure 7 about here.]

\subsubsection{Effect of the truncation thickness}

In order to understand how eigenvalues evolve from the reference case to the $\mathrm{ABH}$ case, intermediate structures are simulated by changing only the truncation thickness. Some examples of thickness profiles are plotted on Fig. 8(a) and results are given on Fig. 8(b) (• in gray levels), completing the results of Fig. 6(b).

From Fig. 8(b), it is clear that the imaginary part of a given eigenvalue decreases with the thickness of the tapered profile. This suggests a small increase in modal density : since the natural frequencies are shifted towards the bottom of the frequency range, there is more modes in a given range.

Another phenomenon is the increase of the real part of the eigenvalues with the decrease of the thickness of the profile, as seen in Sec. 4.1.1. This 
leads to a progressive augmentation of the modal loss factors in most of the cases. An explanation is a greater action of the damping layer at small thicknesses. However, as shown in the work of Georgiev [8], at very small thicknesses the bending stiffness and the added mass of the layer counterbalance its effect on the equivalent material loss factor.

Hyper-damped modes (such as modes 23 and 41 on Fig. 6(b)) appear at lower frequencies with the decrease of the truncation thickness. This lowering effect is much more pronounced for them than for the majority of modes. They add to the rest of the modes in a given frequency band and so have an effect on modal density. As with other modes, the real parts of the associated eigenvalues are moved towards the right of the spectrum, resulting in an increase of their loss factor as the truncation thickness decreases.

[Figure 8 about here.]

\subsection{Mode shapes, localisation and damping}

Fig. 9 shows the modal shapes of modes 14, 23, 41 and 42 that are indicated on Fig. 6(b). The modal shapes are here represented on the transformed grid, on which the problem was solved (see Sec. 3.2). This means that the tapered region is magnified in an adapted manner which is convenient to observe the local behaviour of the $\mathrm{ABH}$. Note that in this case the wavelength appears to be the same everywhere, which is not the case in reality. 
For a given mode $\phi_{n}(x, y)$, the localisation of the kinetic energy is described by the localisation index $I_{k}$ :

$$
I_{k}=\frac{\int_{0}^{b} \int_{0}^{x_{\mathrm{ABH}}} \rho(x) \phi_{k}(x, y)^{2} \mathrm{~d} x \mathrm{~d} y}{\int_{0}^{b} \int_{0}^{L} \rho(x) \phi_{k}(x, y)^{2} \mathrm{~d} x \mathrm{~d} y} .
$$

This indicator is simply the ratio between the kinetic energy in the varying thickness region $\left(0 \leq x \leq x_{\mathrm{ABH}}\right)$ and the total kinetic energy in the structure. The same indicator can be defined for the modes of the reference beam; in this case, it is still the kinetic energy in the region $0 \leq x \leq x_{\mathrm{ABH}}$ that is used. In the reference case, the structure is uniform and the energy of a given mode tends to be uniformly distributed. It implies that the localisation index is low and tends to a constant value for each mode $k$. Results show values around $7 \%$.

Modes 14 and 42 of ABH (Fig. 9(a) and (d)) are typical beam flexural 455 modes. Their localisation index is between 20 and 30\%. The localisation of the energy originates from the higher amplitude and the smaller wavelength. This beam behaviour and this moderate localisation are common to the majority of modes among which modes 14 and 42 lie. Note also that the localisation in this case is higher than that of the uniform beam. The kinetic energy is localised in a region that has a high equivalent material loss factor, according to the Ross-Ungar-Kerwin model; it implies an important energy dissipation and it can be related to an augmentation of the global modal loss factor. 
(see Sec. 4.1.1). They have a strong localisation index (higher than 60\%) meaning they are in fact quasi-local modes. These modes have a locally two dimensional behaviour. One can infer that part of the very high damping of these comes from the extreme localisation, as for modes 14 and 42 . It also comes from the transverse behaviour, since physically it implies more motion of the damping layer and, thus, a higher energy dissipation. The explanation of this transverse behaviour lies in the wavelengths being smaller than the width of the plate in this region. As the beam can be seen as a waveguide, such modes correspond to transverse modes of the guide. Interestingly, the present two dimensional plate model for the $\mathrm{ABH}$ beam shows the limits of one dimensional approaches which are used in the literature $[6,8,9]$ to explain damping induced by $\mathrm{ABH}$.

[Figure 9 about here.]

\subsection{Modal density}

The purpose of this paragraph is to show that the specific tapered profile placed at one end of a otherwise uniform beam can lead to an increase of its modal density, as the experimental result (Fig. 4(a)) and the study of the eigenvalues (Fig. 8) suggest. Two parametric studies are made: the first makes the truncation thickness $h_{t}$ vary while the second makes the exponent $m$ of the profile vary. Results concern conservative structures without damping layer, in order to evaluate the influence of the profile only on the modal density. One has to remind that the damping layer modifies the real part of the flexural rigidity and the mass; it has indeed an influence on the eigenfre- 

densities.

\subsubsection{Effect of the truncation thickness}

Fig. 10 shows the estimated modal densities for the flexural modes of the reference beam and three cases of $\mathrm{ABH}$ profile with different truncation thicknesses. Undoubtedly, there is an increase of modal density for the beams with tapered profiles compared to the reference beam. This increase can be related to the local change in wave speed. While for the reference beam, the mode spacing is proportional to $1 / \sqrt{\omega}$ [31], the repartition of modes is less predictable for the $\mathrm{ABH}$ beam. Moreover, the decrease of the truncation thickness is related to an increase in the modal density, and ultimately, the shape of the result does not have anything to do with the modal density of the uniform beam.

[Figure 10 about here.]

\subsubsection{Effect of the exponent of the power-law defining the thickness profile}

Fig. 11 shows the modal densities for beams with tapered profiles having a different exponent, ranging from 2 to 3 , and the same truncation thickness. A higher exponent gives a profile that is thin over a greater length. From these estimations, it can be concluded that a higher exponent leads to a different modal density, although it is not as obvious as in Fig. 10. This means that the thickness of the extremity really influences the modal density, more than the value of the exponent of the power-law defining the thickness profile. Practically, a real structure will not have its modal density significantly 
increased if the lower limit in thickness is not overcome.

[Figure 11 about here.]

These results allow to conclude that a significant increase in modal density is unlikely to happen with the real damped structures, as was seen in Fig. 4(c).

\section{Modal Overlap Factor and comparison between numerical and experimental results}

The modal overlap factor is a convenient quantity to observe if one wishes to compare the experiment and the model in the framework of a mid or high frequency study. It embeds fine phenomena such as the change in modal density or the damping mechanisms, but allows to globally quantify and qualify the adequateness of the model. Fig. 12 represents the estimated MOF for the experimental reference and $\mathrm{ABH}$ cases and their simulated counterparts. One can remark that the hyper-damped and transverse modes are not represented since experiments did not allow to identify them: they are localised thus non observable using the experiment described in Sec. 2.1 and they induce a MOF far superior to $100 \%$, according to the model. With carefully chosen parameters, one can see very similar tendencies on these plots in the ABH case. Especially interesting are the local maxima around 400, 900 and $2000 \mathrm{~Hz}$ which are present in both the experiment and the simulation; these are likely to come from the variations of the modal loss factor, observed on Fig. 7. Although some of these peaks are localised at the same frequencies 
than hyper-damped modes according to Fig. 7, the two phenomena are not related: a numerical analysis using different structural parameters does not yield matching frequencies; moreover there is no transverse behaviour in the shapes of the modes constituting these small peaks.

Over $3000 \mathrm{~Hz}$, the model fails to reproduce experimental observations. One may think of an imperfect estimation of the experimental parameters. One can also remind that the model uses a very simple structural damping: combined with frequency-independent material loss factors, the Ross-UngarKerwin model is a convenient way to express the effect of the damping layer but does not reflect the complexity of the vibrating layered structure, mainly due to the imperfections, the glueing and the relative importance of the layer versus the structure.

550

This figure shows the general effect of the $\mathrm{ABH}$ on the structure, which is the increase of its MOF. This way to characterize the frequency response is common for mid and high frequency problems. It appears here as a convenient approach to study the $\mathrm{ABH}$ effect on plate-like structure, on which the same fine phenomena take place.

[Figure 12 about here.]

\section{Conclusions}

This paper presents an experimental study of a beam treated with an 560 $\mathrm{ABH}$, as well as a numerical analysis that mostly agrees with the experimen- 
tal results.

Experiments and numerical study show that the $\mathrm{ABH}$ treatment leads to a strong increase of the modal loss factors and a minor increase of the modal

However, experiments on plates show a typical high frequency behaviour and fully justify the type of analysis made in this paper.

[1] D. Ross, E. Ungar, E. Kerwin, Damping of plate flexural vibrations by means of viscoelastic laminae, in: Structural damping, pergamon press 
[2] A. Nashif, D. Jones, J. Henderson, Vibration Damping, A. WileyInterscience Publication, 1985.

[3] M. Géradin, D. Rixen, Mechanical Vibrations: Theory and Applications to Structural Dynamics, John Wiley \& Sons, 1997.

${ }_{590}$ [4] K. Graff, Wave motion in elastic solids, Dover Publications, New York, 1991.

[5] C. Vemula, A. Norris, G. Cody, Attenuation of waves in plates and bars using a graded impedance interface at edges, Journal of Sound and Vibration 196 (1) (1996) 107-127.

[6] V. Krylov, F. Tilman, Acoustic 'black holes' for flexural waves as effective vibration dampers, Journal of Sound and Vibration 274 (2004) 605-619.

[7] V. Krylov, R. Winward, Experimental investigation of the acoustic black hole effect for flexural waves in tapered plates, Journal of Sound and Vibration 300 (2007) 43-49.

[8] V. Georgiev, J. Cuenca, F. Gautier, L. Simon, V. Krylov, Damping of structural vibrations in beams and elliptical plates using the acoustic black hole effect, Journal of Sound and Vibration 330 (2011) 2497-2508.

[9] M. Mironov, Propagation of a flexural wave in a plate whose thickness 605 decreases smoothly to zero in a finite interval, Soviet Physics: Acoustics 34 (3) (1988) 318-319. 
[10] V. Georgiev, J. Cuenca, M. Gautier, F. Moleron, L. Simon, Numerical and experimental investigation of the acoutic black hole effect for vibration damping in beams and elliptical plates, in: Euronoise 2009, Edinburgh, 2009.

[11] F. Gautier, J. Cuenca, V. Krylov, L. Simon, Experimental investigation of the acoustic black hole effect for vibration damping in elliptical plates, in: Proceedings of Acoustic'08, Paris, 2008.

[12] E. Bowyer, D. O'Boy, V. Krylov, J. Horner, Effect of geometrical and material imperfections on damping flexural vibrations in plates with attached wedges of power law profile, Applied Acoustics 73 (2012) 514523.

[13] E. Bowyer, D. O’Boy, V. Krylov, F. Gautier, Experimental investigations of damping flexural vibrations in plates containing tapered indentations of power-law profile, Applied Acoustics 74 (2013) 553-560.

[14] D. O'Boy, V. Krylov, Damping of flexural vibrations in circular plates with tapered central holes, Journal of Sound and Vibration 330 (2011) $2220-2236$.

[15] R. Lyon, R. Dejong, Theory and Application of Statistical Energy Analysis, Butterworth Heinemann, 1995.

[16] J. Berthaut, M. N. Ichchou, L. Jézéquel, Piano soundboard: Structural behavior and numerical and experimental study in the modal range, Applied Acoustics 64 (2003) 1113-1136. 
[17] R. Schmidt, Multiple emitter location and signal parameter estimation, IEEE Transactions on Antennas and Propagation 34 (3) (1986) 276-280.

[18] Y. Hua, T. Sarkar, Matrix pencil method for estimating parameters of exponentially damped/undamped sinusoids in noise, IEEE Transactions on Acoustics Speech and Signal Processing 38 (5) (1990) 814-824.

[19] R. Roy, T. Kailath, ESPRIT Estimation of Signal Parameters via Rotational Invariance Techniques, IEEE Transactions on Acoustics Speech and Signal Processing 37 (7) (1989) 984-995.

[20] J. Laroche, The use of matrix pencil method for the spectrum analysis of musical signal, The Journal of the Acoustical Society of America 94 (4) (1993) 1958-1965.

[21] K. Ege, X. Boutillon, B. David, High-resolution modal analysis, Journal of Sound and Vibration 325 (2009) 852-869.

[22] B. Elie, F. Gautier, B. David, Macro parameters describing the mechanical behavior of classical guitars, The Journal of the Acoustical Society of America 132 (6) (2012) 4013-4024.

[23] D. J. Ewins, Modal Testing, Research Studies Press, 1984.

[24] R. Badeau, B. David, G. Richard, A new pertubation analysis for signal enumeration in rotational invariance techniques, IEEE Transactions on Signal Processing 54 (2) (2006) 450-458.

[25] M. Wax, T. Kailath, Detection of signals by information theoretic cri- 
[31] G. Xie, D. Thompson, C. Jones, Mode count and modal density of structural systems: relationships with boundary conditions, Journal of Sound and Vibration 274 (2004) 621-651.

[32] A. Leissa, Vibration of plates, Acoustical society of America, London, 1993. 
[33] G. C. Cohen, Higher-order numerical methods for transient wave equations, Springer, 2002.

[34] S. Bilbao, Numerical sound synthesis : finite difference schemes and simulation in musical acoustics, John Wiley \& Sons, Hoboken, 2009.

675 [35] K. Arcas, A. Chaigne, S. Bilbao, Sound synthesis of circular plates by finite differences, The Journal of the Acoustical Society of America 123 (2008) 3522.

[36] M. Rao, Recent applications of viscoelastic damping for noise control in automobiles and commercial airplanes, Journal of Sound and Vibration 680 262 (2003) 457-474. 


\section{List of Figures}

1 (a) Polygonal plate including a circular ABH profile; the coordinates of the edges of the plate are $\mathrm{A}(0,0), \mathrm{B}(0,0.8)$, $\mathrm{C}(0.4,0.72), \mathrm{D}(0.28,0.2), \mathrm{O}(0.2,0.6), \mathrm{E}(0.15,0.35)$ and $\mathrm{F}$ $(0.22,0.5) \mathrm{m}$. The radius of the tapered profile centered in $\mathrm{O}$ is $0.06 \mathrm{~m}$. (b) Comparison of the measured cross-mobilities (impact hammer in $\mathrm{E}$ and accelerometer in $\mathrm{F}$ ) of the reference polygonal plate without $\mathrm{ABH}$ profile (thin full line) and the polygonal plate with $\mathrm{ABH}$ profile covered with a thin damping layer (thick full line). . . . . . . . . . . . . . . . 35

2 (a) picture of an ABH extremity of a beam covered with a damping layer (b) scheme of the experimental setup representing the $\mathrm{ABH}$ beam, the accelerometer, the impact hammer and the acquisition system. . . . . . . . . . . 36 Comparison of the measured mobilities of the reference beam (thin full line) and the ABH beam (thick full line). . . . . . . 37

4 Comparison of experimental results between the reference beam $(\square)$ and the ABH beam (o) : (a) Modal densities $n$ and theoretical prediction for flexural waves in a uniform beam according to Ref. [31] (dashed line), (b) modal loss factors $\eta$, (c) Modal Overlap Factor MOF. . . . . . . . . . . . . . . . 38

5 Scheme of an ABH profile at a beam extremity . . . . . . . 39

6 (a) Thickness profile and (b) Spectrum of the complex eigenvalues of $\operatorname{Ref}(\triangle)$ and $\mathrm{ABH}\left(h_{t}=10 \mu \mathrm{m}\right)(\nabla) . \ldots . . . . .40$ Modal loss factor for Ref. $(\triangle)$ and $\mathrm{ABH}(\nabla) \ldots \ldots \ldots . . . .4$

8 (a) Thickness profile and (b) Spectrum of the complex eigenvalues of $\operatorname{Ref}(\triangle), \operatorname{ABH}\left(h_{t}=10 \mu \mathrm{m}\right)(\nabla)$ and intermediate structures, gradually thinned, between Ref and $\mathrm{ABH}(\bullet$ in gray levels). . . . . . . . . . . . . . . . . . . . . . 42

9 Mode shapes (modulus) and localisation indicator for modes (a) 14, (b) 23, (c) 41 and (d) 42 in the ABH case. The ABH region is indicated by $\tilde{x}_{\mathrm{ABH}}$. The axial coordinate is the transformed coordinate $\tilde{x}$ defined by Eq. 16 . . . . . . . . . . .

10 Numerical modal density for the reference beam (thick full line) and $\mathrm{ABH}$ beams with different truncation thicknesses: $10 \mu \mathrm{m}$ (thin full line), $5 \mu \mathrm{m}$ (dashed line) and $1 \mu \mathrm{m}$ (dotted line), and an exponent of $m=2 \ldots \ldots \ldots$. . . . . . . 4 
11 Numerical modal density for the reference beam (thick full line) and $\mathrm{ABH}$ beams with different exponents: $m=2$ (thin full line), 2.5 (dashed line) and 3 (dotted line), and a trunca-

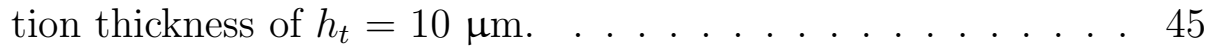

12 Simulated MOF for $\operatorname{Ref}(\triangle)$ and $\mathrm{ABH}(\nabla)$. For comparison, experimental MOF for $\operatorname{Ref}(\square)$ and $\mathrm{ABH}(\circ) . \ldots . . . . .46$ 


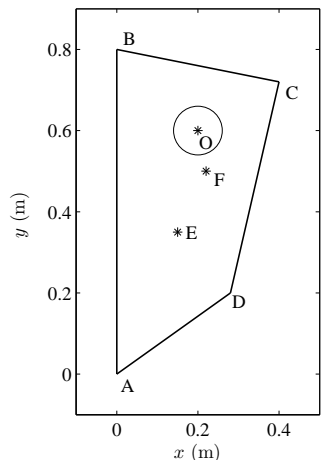

(a)

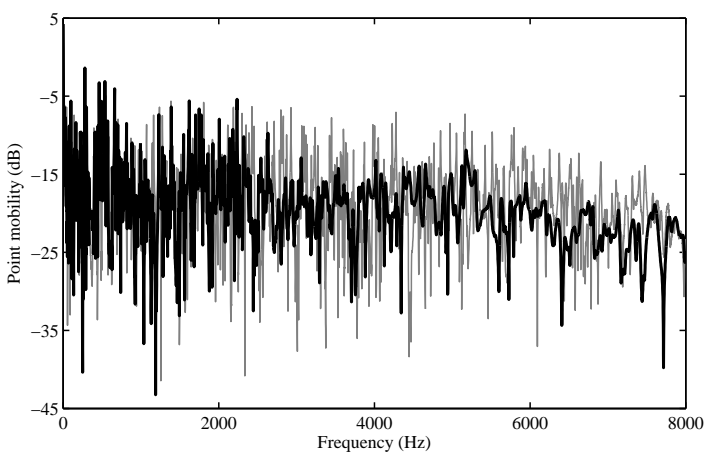

(b)

Figure 1: (a) Polygonal plate including a circular ABH profile; the coordinates of the edges of the plate are A $(0,0), \mathrm{B}(0,0.8), \mathrm{C}(0.4,0.72), \mathrm{D}(0.28,0.2), \mathrm{O}(0.2,0.6), \mathrm{E}(0.15,0.35)$ and $\mathrm{F}(0.22,0.5) \mathrm{m}$. The radius of the tapered profile centered in $\mathrm{O}$ is $0.06 \mathrm{~m}$. (b) Comparison of the measured cross-mobilities (impact hammer in $\mathrm{E}$ and accelerometer in $\mathrm{F}$ ) of the reference polygonal plate without $\mathrm{ABH}$ profile (thin full line) and the polygonal plate with $\mathrm{ABH}$ profile covered with a thin damping layer (thick full line). 


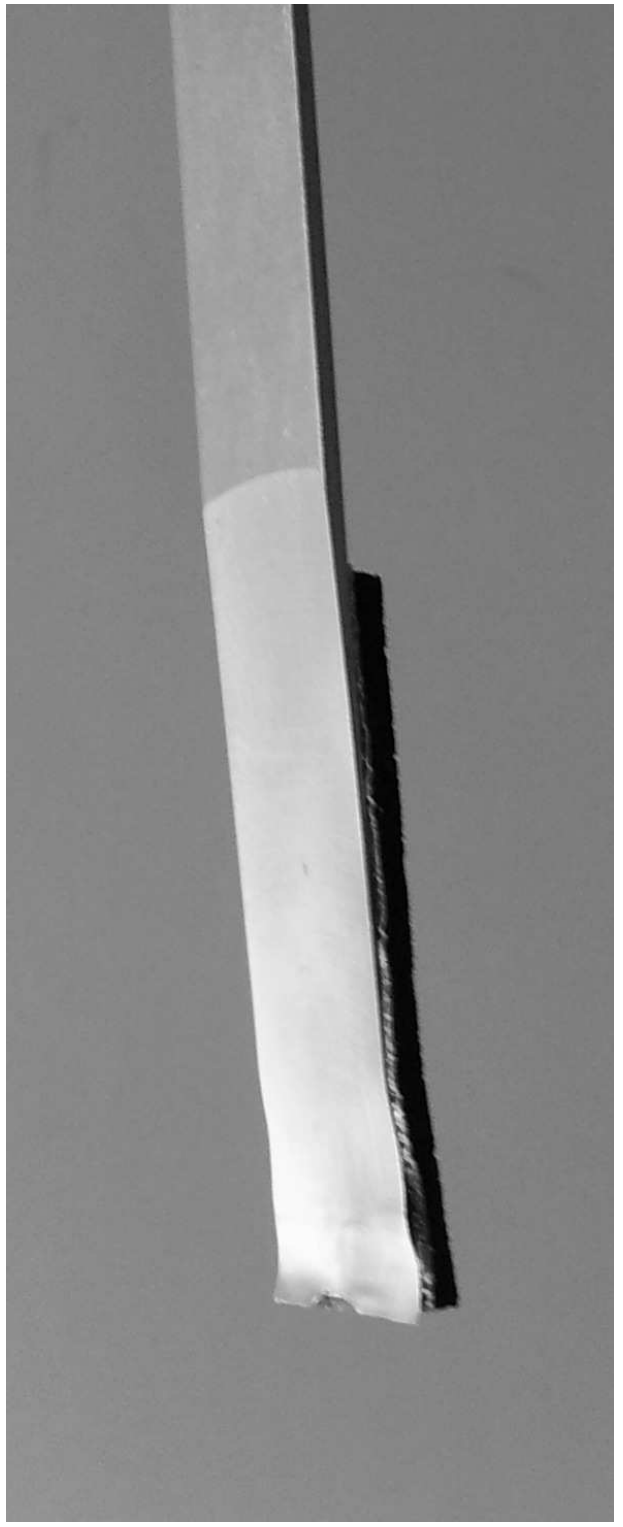

(a)

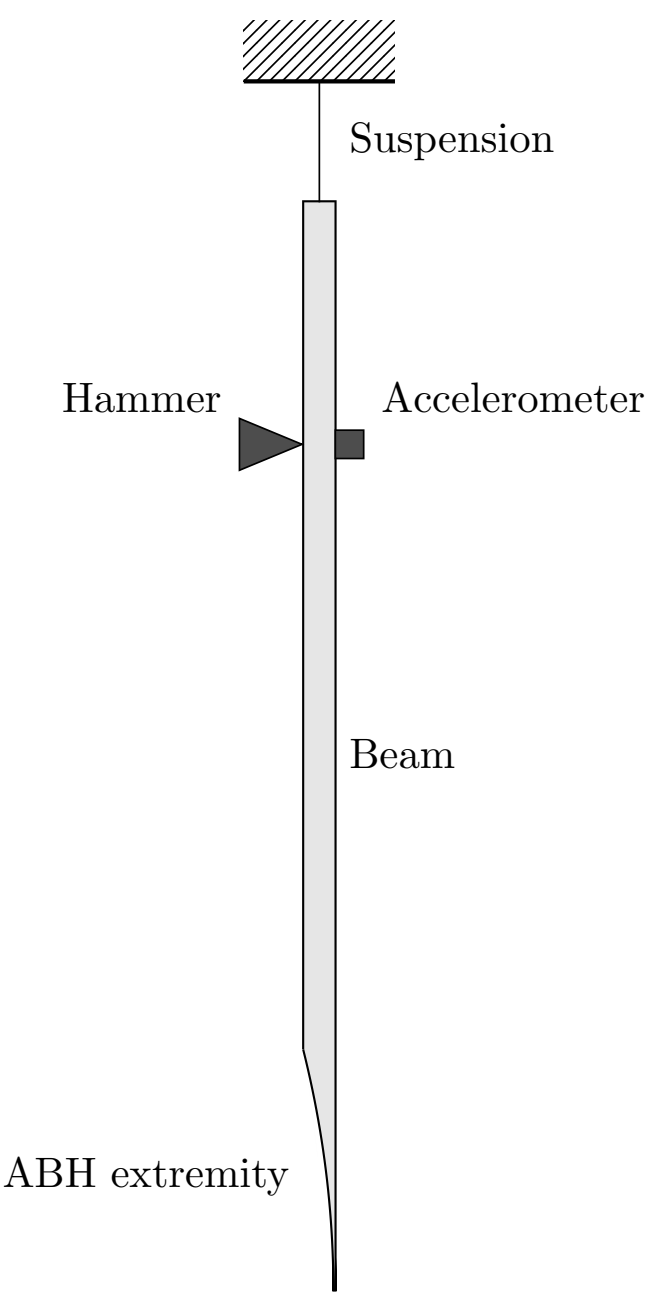

(b)

Figure 2: (a) picture of an ABH extremity of a beam covered with a damping layer (b) scheme of the experimental setup representing the $\mathrm{ABH}$ beam, the accelerometer, the impact hammer and the acquisition system. 


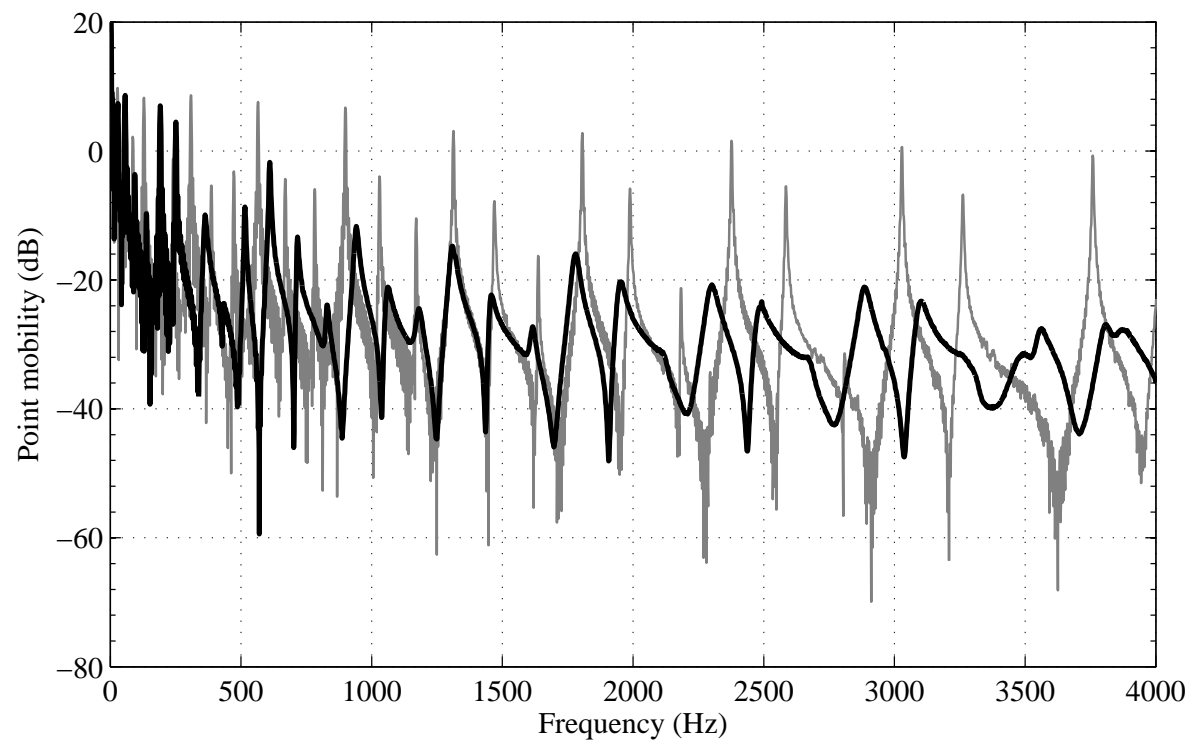

Figure 3: Comparison of the measured mobilities of the reference beam (thin full line) and the ABH beam (thick full line). 


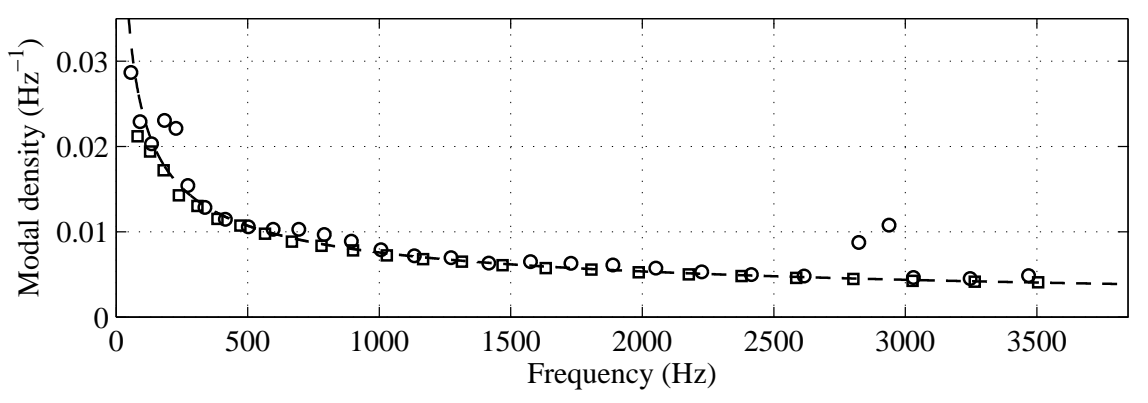

(a)

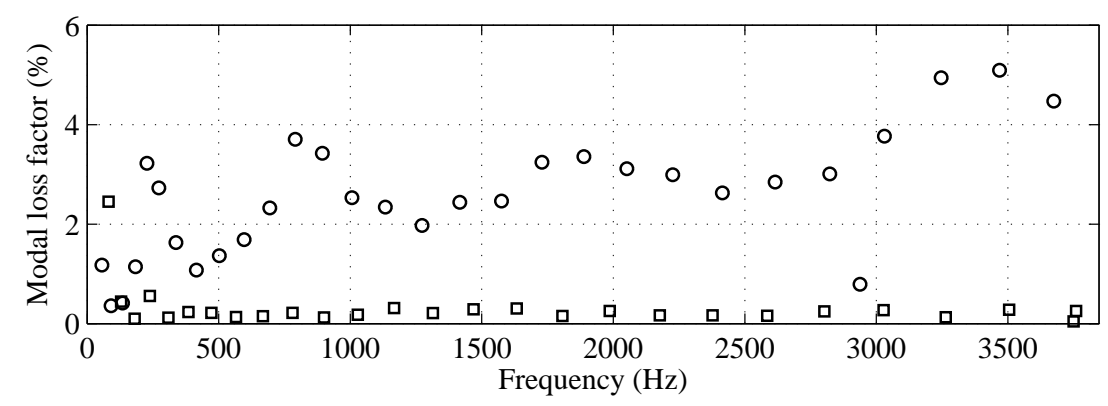

(b)

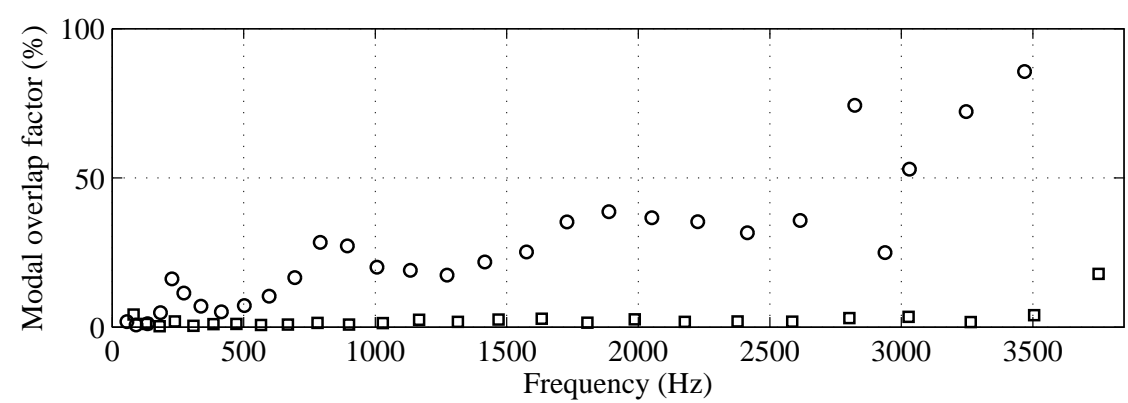

(c)

Figure 4: Comparison of experimental results between the reference beam ( $\square$ ) and the ABH beam (o) : (a) Modal densities $n$ and theoretical prediction for flexural waves in a uniform beam according to Ref. [31] (dashed line), (b) modal loss factors $\eta$, (c) Modal Overlap Factor MOF. 


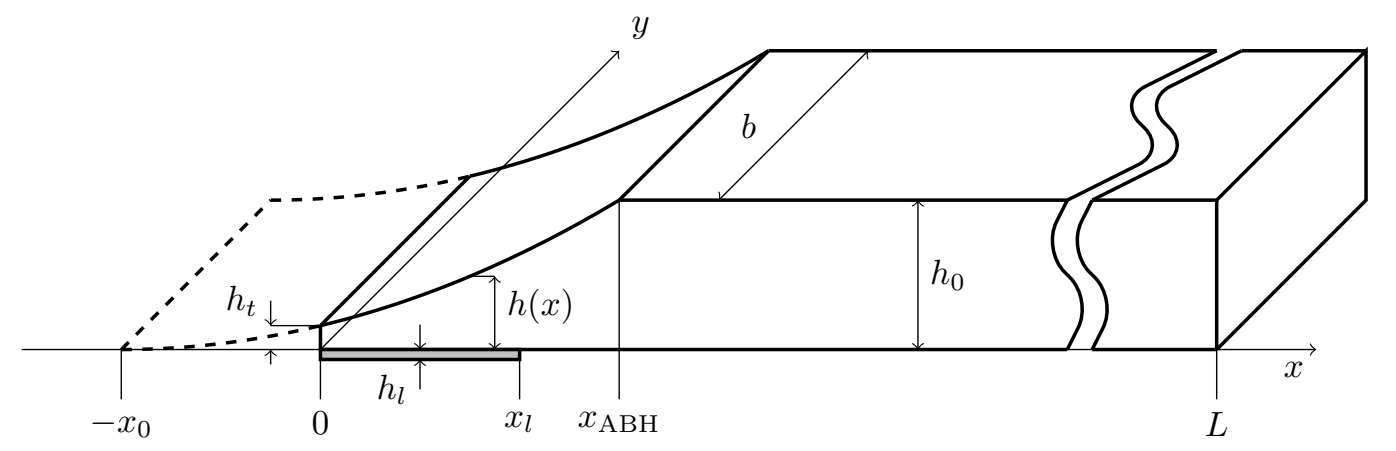

Figure 5: Scheme of an ABH profile at a beam extremity 


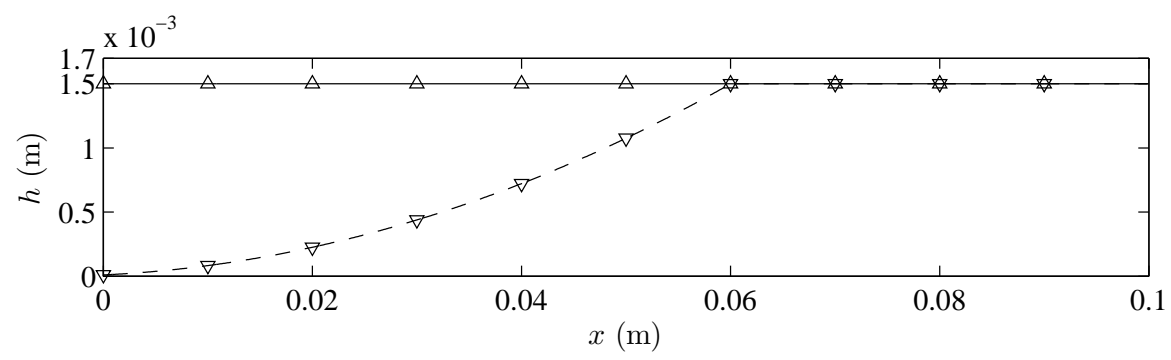

(a)

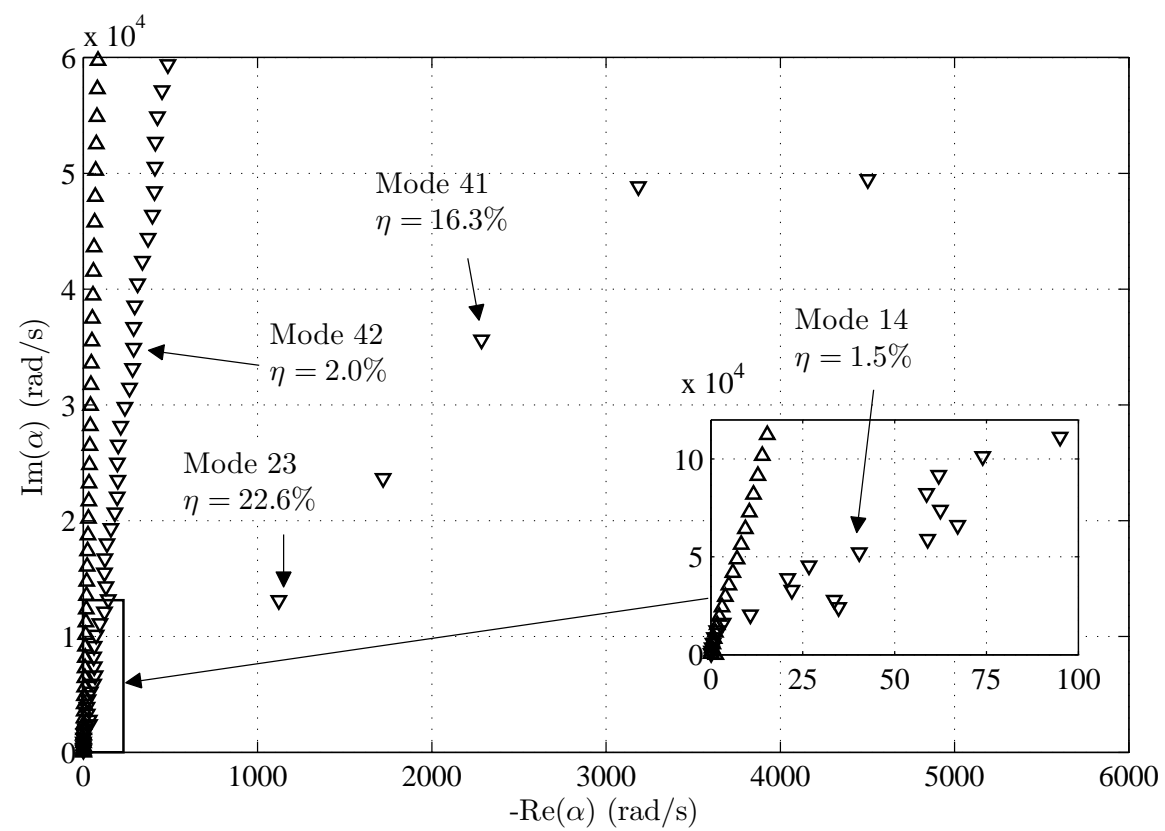

(b)

Figure 6: (a) Thickness profile and (b) Spectrum of the complex eigenvalues of Ref $(\triangle)$ and $\mathrm{ABH}\left(h_{t}=10 \mu \mathrm{m}\right)(\nabla)$. 


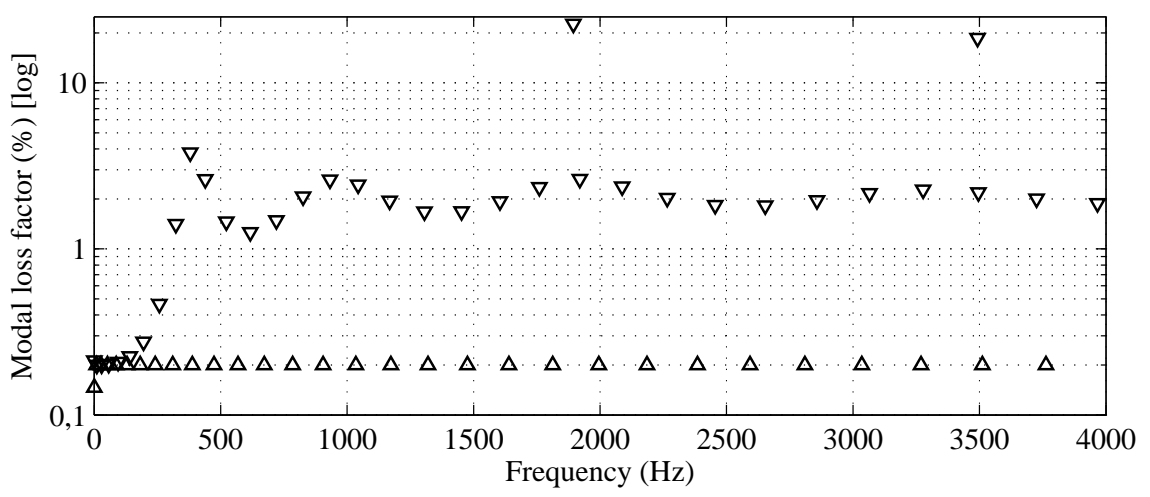

Figure 7: Modal loss factor for Ref. $(\triangle)$ and ABH $(\nabla)$. 


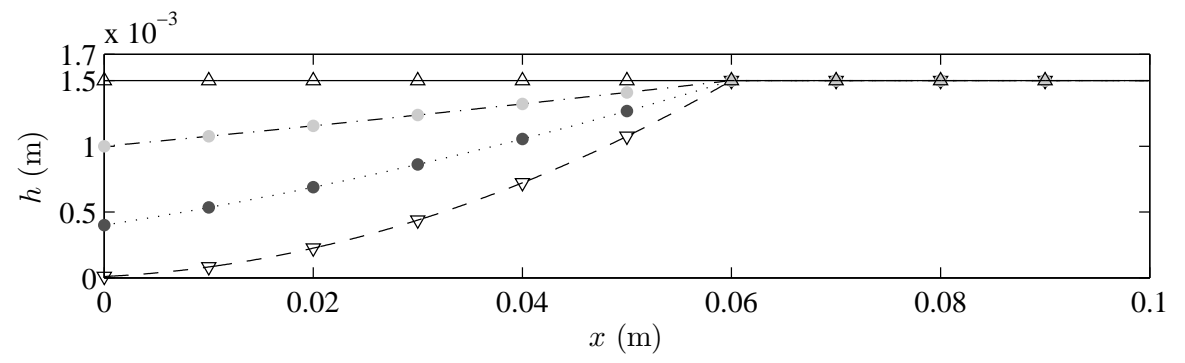

(a)

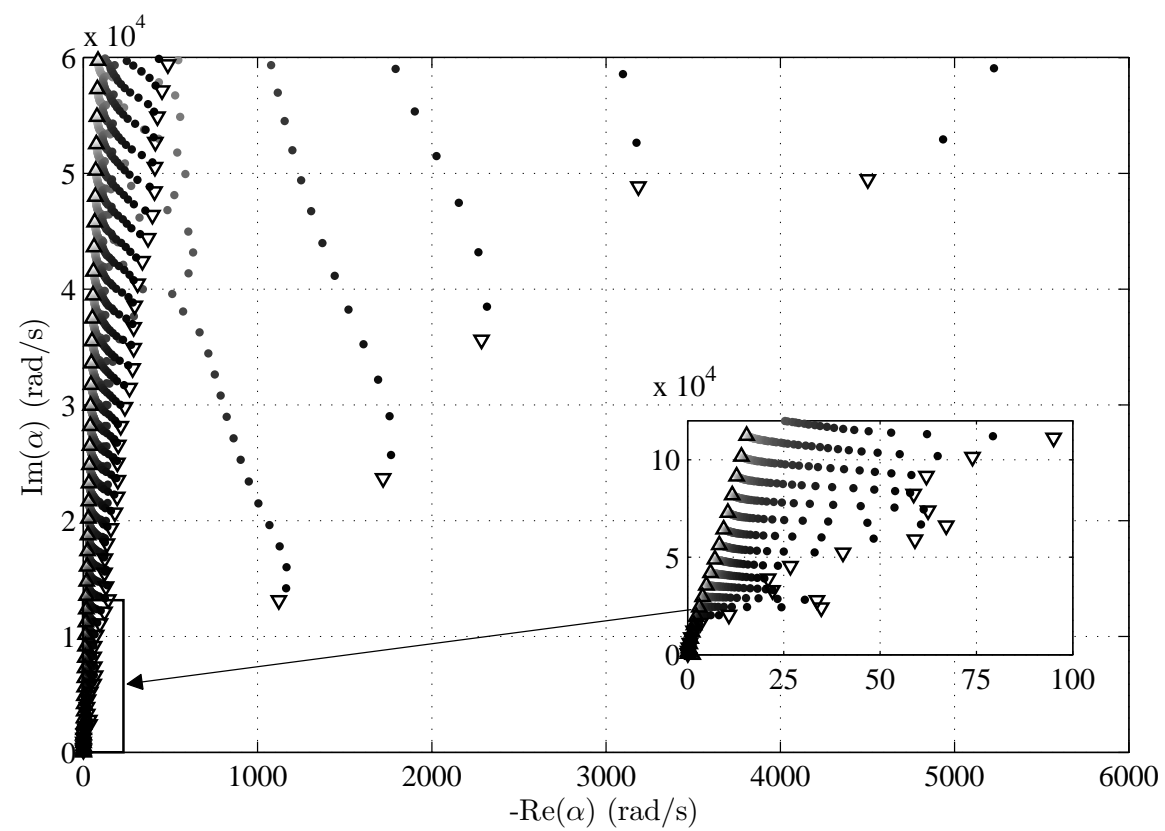

(b)

Figure 8: (a) Thickness profile and (b) Spectrum of the complex eigenvalues of Ref $(\triangle)$, $\mathrm{ABH}\left(h_{t}=10 \mu \mathrm{m}\right)(\nabla)$ and intermediate structures, gradually thinned, between Ref and $\mathrm{ABH}(\bullet$ in gray levels). 


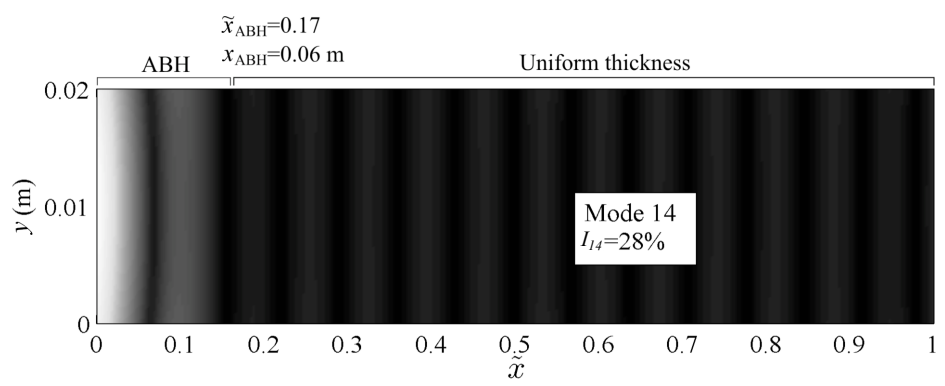

(a)

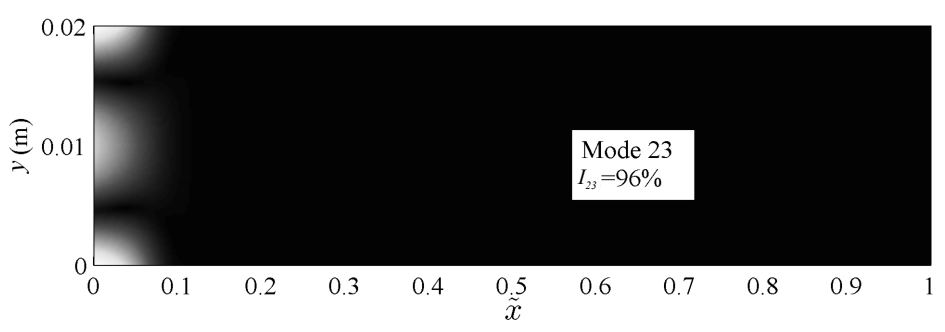

(b)

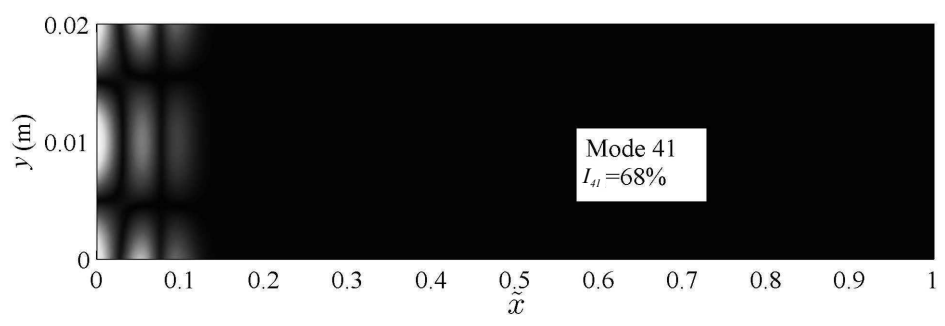

(c)

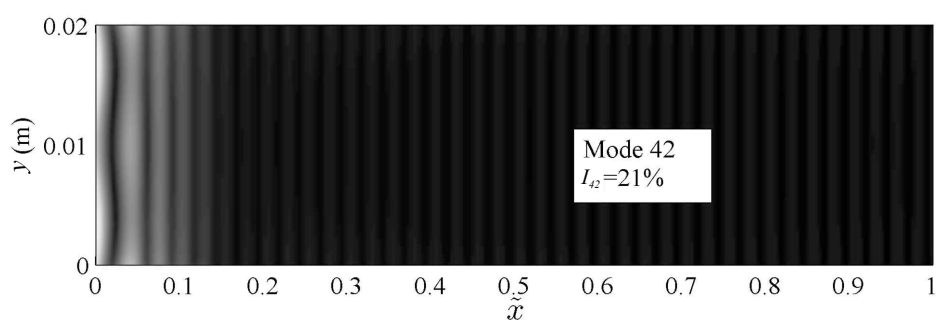

(d)

Figure 9: Mode shapes (modulus) and localisation indicator for modes (a) 14, (b) 23, (c) 41 and (d) 42 in the $\mathrm{ABH}$ case. The $\mathrm{ABH}$ region is indicated by $\tilde{x}_{\mathrm{ABH}}$. The axial coordinate is the transformed coordinate $\tilde{x}$ defined by Eq. 16 . 


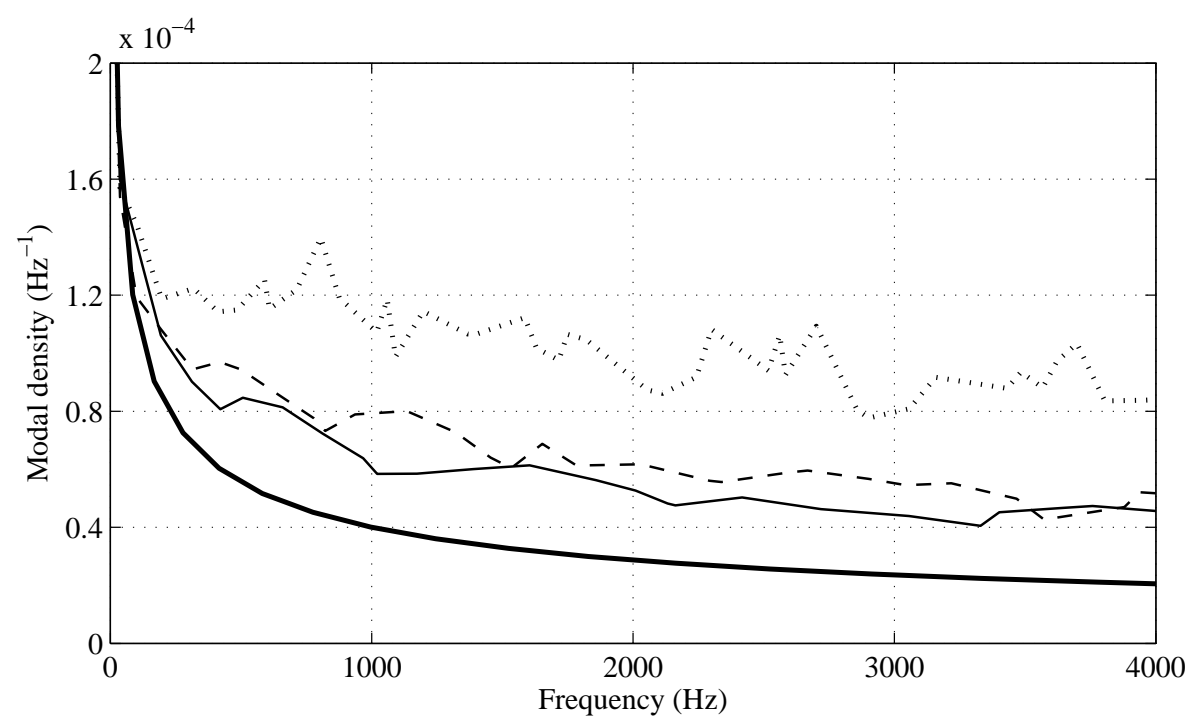

Figure 10: Numerical modal density for the reference beam (thick full line) and ABH beams with different truncation thicknesses: $10 \mu \mathrm{m}$ (thin full line), $5 \mu \mathrm{m}$ (dashed line) and $1 \mu \mathrm{m}$ (dotted line), and an exponent of $m=2$. 


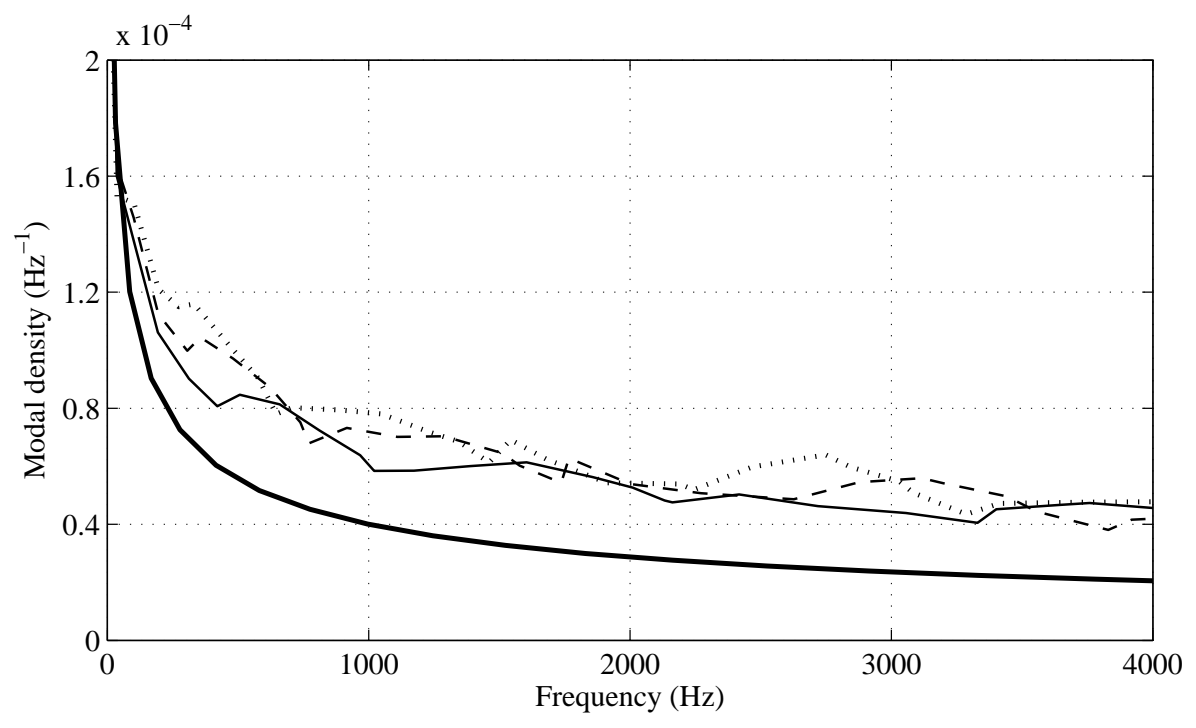

Figure 11: Numerical modal density for the reference beam (thick full line) and ABH beams with different exponents: $m=2$ (thin full line), 2.5 (dashed line) and 3 (dotted line), and a truncation thickness of $h_{t}=10 \mu \mathrm{m}$. 


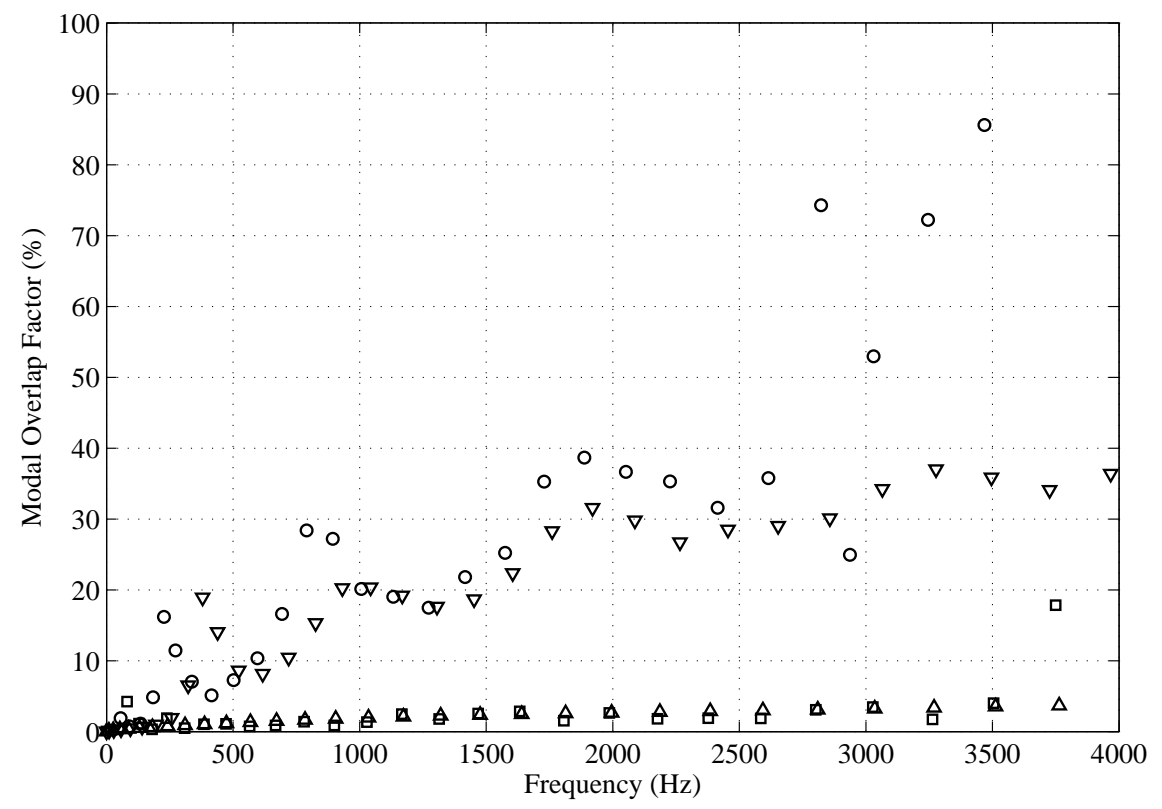

Figure 12: Simulated MOF for $\operatorname{Ref}(\triangle)$ and ABH $(\nabla)$. For comparison, experimental MOF for Ref $(\square)$ and $\mathrm{ABH}(\circ)$. 


\section{List of Tables}

1 Values of the parameters for both the experimental study and the numerical model. . . . . . . . . . . . . . . . . . . . . . 48 
Table 1: Values of the parameters for both the experimental study and the numerical model.

\begin{tabular}{ccccccc}
\hline $\begin{array}{l}\text { Parameters of the beam } \\
E_{p}[\mathrm{GPa}]\end{array}$ & $\rho_{p}\left[\mathrm{~kg} \mathrm{~m}^{-3}\right]$ & $\nu$ & $\eta_{p}[\%]$ & $h_{0}[\mathrm{~mm}]$ & $L[\mathrm{~m}]$ & $b[\mathrm{~mm}]$ \\
\hline 69.8 & 2700 & 0.3 & 0.2 & 1.5 & 0.895 & 20 \\
\hline
\end{tabular}

Parameters of the ABH

\begin{tabular}{ccccc}
$x_{0}[\mathrm{~mm}]$ & $x_{1}[\mathrm{~mm}]$ & $h_{t}[\mu \mathrm{m}]$ & $m$ & \\
\hline 5.3 & 6 & 10 & 2 & \\
\hline Parameters of the damping layer \\
$E_{l}[\mathrm{GPa}]$ & $\rho_{l}\left[\mathrm{~kg} \mathrm{~m}^{-3}\right]$ & $\eta_{l}[\%]$ & $l_{l}[\mathrm{~m}]$ & $h_{l}[\mu \mathrm{m}]$ \\
\hline 7 & 1000 & 40 & 0.05 & 100 \\
\hline \multicolumn{5}{c}{} \\
\hline
\end{tabular}

